\title{
Three day oral course of Augmentin to treat chancroid
}

\author{
J O NDINYA-ACHOLA, ${ }^{*}$ H NSANZE, ${ }^{*}$ P KARASIRA, ${ }^{*}$ L FRANSEN, ${ }^{*} \ddagger$ L J D’COSTA, $†$ P PIOT, $\ddagger$ AND A R \\ RONALD $\S$
}

From the *Department of Microbiology, University of Nairobi, $\uparrow$ Special Treatment Clinic, Nairobi City Council, Nairobi, Kenya, $\ddagger$ Department of Microbiology, Institute of Tropical Medicine, Antwerp, Belgium, and §Department of Medical Microbiology, University of Manitoba, Winnipeg, Canada

SUMMARY Amoxycillin and clavulanic acid (Augmentin; Beecham Research Laboratories) was used to treat patients with bacteriologically proved chancroid in three different dose regimens. A single dose of Augmentin (amoxcycillin $3 \mathrm{~g}$, clavulanic acid $350 \mathrm{mg}$ ) was found to be ineffective. A similar dose repeated after 24 hours was equally ineffective, but a dose (amoxcycillin $500 \mathrm{mg}$, clavulanic acid $250 \mathrm{mg}$ ) given every 8 hours for three days was found to be effective. The drug was well tolerated and no side effects were noted in any of the patients treated.

\section{Introduction}

Chancroid is a common genital ulcer disease in many developing countries. Though the disease is not often seen in the developed countries at the moment, sporadic outbreaks of infection have been recorded from these regions. ${ }^{1}$ In Kenya chancroid accounts for up to $80 \%$ of all genital ulcer disease.

Haemophilus ducreyi, the causative agent of chancroid, has become resistant to the traditionally used drugs, such as tetracycline and sulphonamides. Several clinical trials have shown that other drugs are effective over three to seven days. Some drugs, such as rifampicin, third generation cephalosporins, spectinomycin, and trimethoprim and sulphamatrole, have been used effectively in shorter courses, including single doses (Ronald AR et al and Ndinya-Achola JO et al, abstracts, Third Regional Congress of the African Union of Venereal Diseases and Treponematoses, Nairobi, Kenya, 1983). ${ }^{23}$ In a previous study, treatment of chancroid with amoxycillin and clavulanic acid (Augmentin; Beecham Research Laboratories) for seven days yielded cure rates of over $90 \%$, and $H$ ducreyi was eradicated from the ulcers. ${ }^{4}$

As a shorter course of amoxycillin and clavulanic acid would be advantageous in terms of cost of treatment and patient compliance, we investigated three shorter regimens to treat chancroid.

Address for reprints: Dr J O Ndinya-Achola, Department of Medical Microbiology, University of Nairobi, P O Box 30588, Nairobi, Kenya

Accepted for publication 21 July 1985
Patients, materials, and methods

We recruited into the study male patients seen at the special treatment clinic in Nairobi if they had a genital ulcer after sexual exposure. The cause of genital ulceration was identified in each patient, and only those who were dark field negative and had clinical and microbiological diagnoses of chancroid (positive culture for $H$ ducreyi) were included in the study. Those who had received antimicrobial treatment in th preceding week were excluded.

We examined the ulcers for size, depth, undermining, induration, and tenderness. The number of ulcers was noted and the ulcers were measured.

Culture for $H$ ducreyi was performed using two media: one consisted of gonococcal agar base with $2 \%$ bovine haemoglobin (Gibco, Madison, Wisconsin, USA) and 5\% fetal calf serum (GCHBS), and the other Mueller-Hinton agar base (BBL Division, Becton Dickinson Co, Cockerysville, Maryland, United States of America) with 5\% chocolated horse blood (MHHB). Each had added IsoVitalex (BBL) and vancomycin $3 \mathrm{mg} / \mathrm{l}$ as described by Nsanze and others. $^{5}$

Urethral cultures for Neisseria gonorrhoeae were performed in patients who had a urethral discharge. Specimens for herpes simplex studies were taken in virus transport medium and examined by tissue culture and direct immunofluorescence. Dark ground microscopy was performed in all cases on the first day of attendance. Blood was taken for syphilis serology from all patients at the initial visit and 10 days later.

Patients who satisfied the criteria for inclusion in 
the study were treated with one of three dose regimens. In the first regimen Augmentin was given as a single dose (amoxycillin $3 \mathrm{~g}$ and clavulanic acid $350 \mathrm{mg}$ ), in the second the single dose was repeated after 24 hours, and in the third Augmentin was given at a dose of amoxycillin $500 \mathrm{mg}$ and clavulanic acid $250 \mathrm{mg}$ every eight hours for three days.

The patients were seen again on days $3,7,10$, and 14 after the initial treatment. During the follow up visits the ulcers were assessed as on the first day, and culture for $H$ ducreyi was repeated if the ulcer had not healed. Cure was defined by complete epithelialisation of all ulcers. . Cure rates were calculated as a percentage using the formula:

No newly healed + No healed previously

No seen + No healed previously $\times 100$.

\section{Results}

We treated 10 patients using the first regimen. Two did not attend adequate follow up visits. Of the remaining eight, only one patient's ulcers became bacteriologically negative and eventually progressed to cure. The remaining seven remained culture positive even up to the seventh day after treatment, and the ulcers showed little or no signs of healing.

We treated 12 patients using the second regimen. Two were excluded from analysis because of inadequate follow up. Cure was achieved in three patients. Seven remained culture positive with persistent ulcers and were regarded as treatment failures.

We enrolled 63 patients for treatment using the third regimen. Their ages ranged from 16 to 48 , but most were aged between 20 and 30 . Of the 63 patients only 44 were treated, as 19 were excluded from further analysis because they had dark field positive ulcers or $H$ ducreyi culture negative ulcers or because they failed to attend for treatment. All ulcers had diameters of 6$20 \mathrm{~mm}$ except for those in six patients, three of whom had ulcers of $5 \mathrm{~mm}$ or less in diameter and three had ulcers with diameters greater than $20 \mathrm{~mm}$ (table). The mean time to healing of the ulcers was proportional to their size at presentation.

The figure shows that cure rates were $24 \%, 57 \%$, $73 \%$, and $94 \%$ on days $3,7,10$, and 14 respectively.

Eight patients had buboes. In all eight healing occurred without the buboes progressing to fluctuation.

Treatment failed in two patients receiving the third regimen. Both had ulcers that regressed initially and were culture negative for $H$ ducreyi on the third day, but by the seventh day after treatment the ulcers had increased in size and reverted to culture positivity. One of these patients had an ulcer with an initial diameter of $12 \mathrm{~mm}$, and during the course of follow up no other abnormality was noted. He was successfully treated using a different treatment. The second patient had an

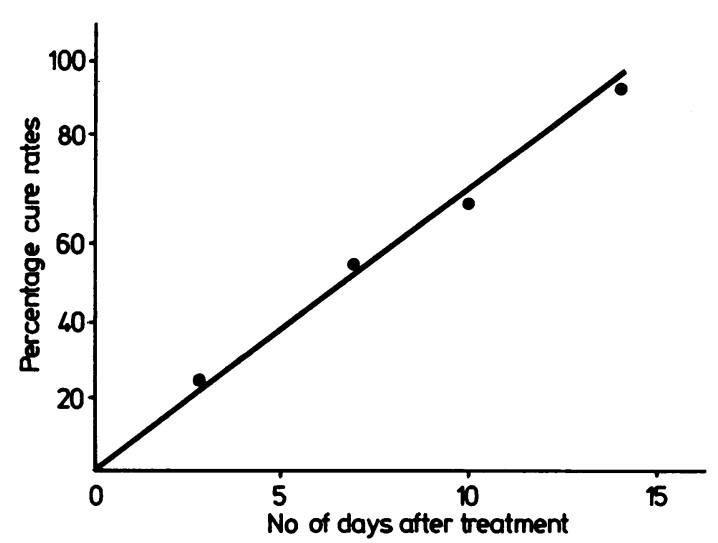

FIGURE Cure rates seen on various follow up days in 44 patients with chancroid treated with Augmentin (amoxycillin $500 \mathrm{mg}$ and clavulanic acid $250 \mathrm{mg}$ ) every eight hours for three days.

ulcer that was initially $9 \mathrm{~mm}$ in diameter. He also had an improvement in his symptoms initially. When it was noted that he was not improving further with this regimen, he was given a second course of treatment. At ' day 10 after treatment the ulcer was still culture positive for $H$ ducreyi. At day 14 he developed generalised lymph node enlargement and complained of evening fevers. Investigations including peripheral blood examination and chest $x$ rays showed no abnormality, and he was lost to follow up before any further investigations could be carried out. Treatment with two other regimens had also failed, and he may have had an underlying disease affecting the immune system.

\section{Discussion}

Antimicrobial chemotherapy against chancroid has attracted much attention recently. The occurrence of relatively high failure rates with sulphonamides and tetracyclines has increased the need to find new chemotherapeutic agents effective against this disease. ${ }^{6}$ In Kenya, a number of drugs including erythromycin, rifampicin, trimethoprim/sulpha-

TABLE Average ulcer size and healing time in 44 patients with chancroid treated with Augmentin (amoxycillin $500 \mathrm{mg}$ and clavulanic acid $250 \mathrm{mg}$ ) every eight hours for three days

\begin{tabular}{lcc}
\hline $\begin{array}{c}\text { Diameter of } \\
\text { largest ulcer }(\mathrm{mm})\end{array}$ & $\begin{array}{c}\text { No of } \\
\text { patients }\end{array}$ & $\begin{array}{c}\text { Mean time to } \\
\text { healing (days) }\end{array}$ \\
\hline $2-5$ & 3 & $4 \cdot 3$ \\
$6-10$ & 21 & $6 \cdot 6$ \\
$11-15$ & 14 & $6 \cdot 9$ \\
$16-20$ & 3 & $7 \cdot 3$ \\
20 & 3 & $10 \cdot 3$ \\
\hline
\end{tabular}


metrole, cefotaxime, and ceftriaxone have all been shown to be effective in the treatment of chancroid.

The safety of penicillins is a major advantage for this class of antibiotics. In chancroid, however, the use of amoxycillin alone is greatly restricted because of $B$ lactamase production. In Kenya, as in South Africa and South East Asia, most strains of $H$ ducreyi produce $B$ lactamase. ${ }^{78}$ For the successful use of amoxycillin in treating chancroid, this antibiotic needs to be protected against $B$ lactamase. Clavulanic acid has been shown to do this both in vitro and in vivo. ${ }^{9}$

In a previous study a seven day course of amoxycillin and clavulanic acid was shown to be effective treatment for chancroid. ${ }^{4}$ The applicability of this seven day regimen in developing nations is limited by expense and problems with patient compliance. The present study shows that a single dose of amoxycillin and clavulanic acid or two doses at 24 hour intervals are both ineffective for treating chancroid. The three day course has been found to be effective, and the cure rates compare favourably with those obtained using other regimens. It was well tolerated by all the patients, and no side effects were reported. The severe manifestations of chancroid, namely large ulcers and buboes, also responded favourably to the treatment, though there was a progressive increase in mean healing time with size of ulcers.

We conclude that the three day amoxycillin and clavulanic acid regimen used in this study is safe and effective treatment for chancroid.
This study was supported by funds from the World Health Organisation Beecham Pharmaceuticals and carried out under the auspices of the WHO collaborative centre for sexually transmitted disease research and training, Nairobi, Kenya.

\section{References}

I Hammond GW, Slutchuk M, Scatliff J, Sherman E, Wilt JC, Ronald AR. Epidemiologic, clinical, and therapeutic features of an outbreak of chancroid in North America. Rev Infect Dis 1980;2:867-79.

2 Plummer F, Nsanze H, D'Costa LJ, et al. Short course and single dose antimicrobial therapy for chancroid in Kenya: reports of studies with rifampin combinated with trimethoprim and rifampin alone. Rev Infect Dis 1983;5:5565-72.

3 Plummer FA, Nsanze H, D'Costa LJ, et al. Single-dose therapy of chancroid with trimethoprim-sulfametrole. $N$ Engl $J$ Med 1983;309:67-71.

4 Fast MV, Nsanze H, D'Costa LJ, et al Treatment of chancroid by clavunalic acid with amoxycillin in patients with $\beta$ lactamase-positive Haemophilus ducreyi infection. Lancet 1982;ii:509-11.

5 Nsanze H, Plummer F, Maggwa ABN, et al. Comparison of media for the primary isolation of Haemophilus ducreyi. Sex Transm Dis 1984;11:1-6.

6 Fast MV, Nsanize H, D'Costa LJ, et al. Antimicrobial therapy of chancroid: an evaluation of five treatment regimens correlated with vitro sensitivity. Sex Transm Dis 1983;10:1-6.

7 Nsanze H, Fast MV, D'Costa LJ, Tukei P, Curan J, Ronald AR. Genital ulcers in Kenya: a clinical and laboratory study. British Journal of Venereal Diseases 1981;57:378-81.

8 Bilgeri YN. A microbiological study of chancroid in Johannesburg. Johannesburg: University of Witwaterstand, 1983. (MSc Thesis.)

9 Beale AS, Comber KR, White AR, Sutherland R. First symposium on Augmentin (clavulanate potentiated amoxycillin). Amsterdam: Excerpta Medica, 1981:127. (International Congress Series, No 554.) 